\title{
Multi-sensing based target tracking by using decision-making strategy with spatial and temporal properties
}

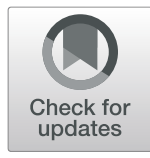

\author{
Liu Yang ${ }^{1}$, Liu Xiuju ${ }^{2 *}$, Jin Huixia', Fu Yuanyuan ${ }^{1}$ and Zhang Chi ${ }^{1}$
}

\begin{abstract}
Multi-sensing system for target tracking has been addressed by many researchers from different fields. In this work, a multi-sensing system within the time, space, and frequency domains is first described. The system development is based on sensor spatial and temporal characteristics, and therefore is reliable and stable. The frequency outcome of sensors is employed to segment the detection scope into different domains, and is simple to implement. For resolving the challenge of decision-making strategy, this work combines the spatial and temporal properties with the determination mechanism as well as optimizes the system devising - making it a very promising basis for the realization of tracking accuracy improvement. With this strategy, target detection results are obtained and evaluated by probability-based parameters. The proposed decision-making scheme receives a high detection accuracy as well as a good working performance according to statistical analysis, allowing a straightforward methodology for the configuration of multi-sensing system.
\end{abstract}

Keywords: Multi-sensing system, Target tracking, Spatial and temporal characteristics, Decision-making strategy

\section{Introduction}

The significance of sensors for target tracking as well as its application has attracted a great deal of interest over last decades. With the evolution of sensing technology, the sensing platforms have become increasingly complex [1]. As such, more information from sensors is gained to improve tracking reliability and accuracy. Nevertheless, the demand of signal detecting results in a big volume and a low speed of tracking [2]. This problem is most pronounced in the difficulties in real-time tracking and object analysis. The utilization of multi-sensing system, which is featured by combing tracking outputs with multiple resources, provides users with comprehensive and complementary information [3]. Thereby, the information from multiple sensors can be integrated to gain a better understanding of tracking.

On the basic of previous researches, multi-sensors have already been employed for traffic speed and travel time detecting [4-6]. Indoor positioning is another such field, with recent publications revealing the capability in

\footnotetext{
* Correspondence: exiu0824@126.com

${ }^{2}$ College of Computer, Heze University, Heze 274000, Shandong, China

Full list of author information is available at the end of the article
}

healthcare monitoring $[7,8]$, surveillance $[9,10]$, and target group pattern generation [11, 12]. Multi-sensing systems outperform traditional tracking techniques in managing the flow of signal and coordinating sensor actions [13]. A tracking system equipped with sensors, which functions in an autonomous or semi-autonomous mode, has to be capable of defining the target from the sensors as well as making decisions based on this information [14]. The information of different modalities can be applied to obtain an exact understanding of the target with signal integration. In a multi-sensing system, multiple sensors inevitably introduce problems of data redundancy or data conflicting due to the data stored in multiple databases with inconsistent attributes [15-17]. The use of available data, the connectivity and diversity between data sources, and abilities of data analytic methods make the tracking precision still a challenge. Without the strategies of precise positioning, the address of sensor output can hardly be reliable. That is, the accuracy improvement of sensing system is identified as a specific issue in tracking optimization. The importance of this issue is that it is an underlying concern of the sensing system. 
Many techniques have been explored in the improvement of tracking accuracy. Previously, Alon Shalev Housfater applied the idea of sequential Monte Carlo method to nonlinear inputs optimization in multi-sensor tracking system [18]. Their work indicates that the Monte Carlo algorithm can rapidly converge to get an optimal result. Somnath Deb et al. proposed an S-dimensional assignment algorithm, provided as a more effective alternative, in sensor outputs association and estimation in surveillance [19]. For indoor tracking, combination of Kalman sensor group fusion architecture and Alpha-Beta filter is devised to handle persons randomly walking and positioning issues [20]. Recent research works face this problem by implementing processing algorithms in the presence of multi-sensing systems.

In this paper, we will consider the decision-making mechanism of tracking. A set of decision-making strategies is proposed for accuracy improvement. Accordingly, the remaining of this paper is organized as follows: the problem statement as well as the basic working model is presented in the next section. The decision-making strategy in target tracking is proposed in Section 3 followed with the mathematical analysis. The signal processing principle is depicted in brief and the experimental outcomes for test the proposed methodology and data analysis results are shown in Section 4. Conclusions with discussion are given in the last section.

\section{Basic concepts on spatial and temporal characteristics}

We describe now the theory of spatial and temporal features of moving objects, which influences the accuracy demanded of tracking [21]. Within this research, the characteristics integration refers to the synergistic use of the both spatial and temporal information provided by multiple sensory devices to accomplish the tracking task. Let

$$
R=\{T, S, f\}
$$

be a three-dimension domain, with $T$ standing for time domain, $S$ for spatial domain, and $f$ for frequency domain. During a specific time interval, the occupation area of the sensing node is computationally efficient to work out with positioning coordinates $D_{x}$ and $D_{y}$. Then the area of one single sensor is presented as follows:

$$
S_{R}=D_{x} \times D_{y}
$$

Hence, each sensor is normalized and defined in the three-dimension domain as shown in Fig. 1.

On the foundation of research [22], the object is characterized with remarkable time and spatial characteristics under moving condition. One of the key

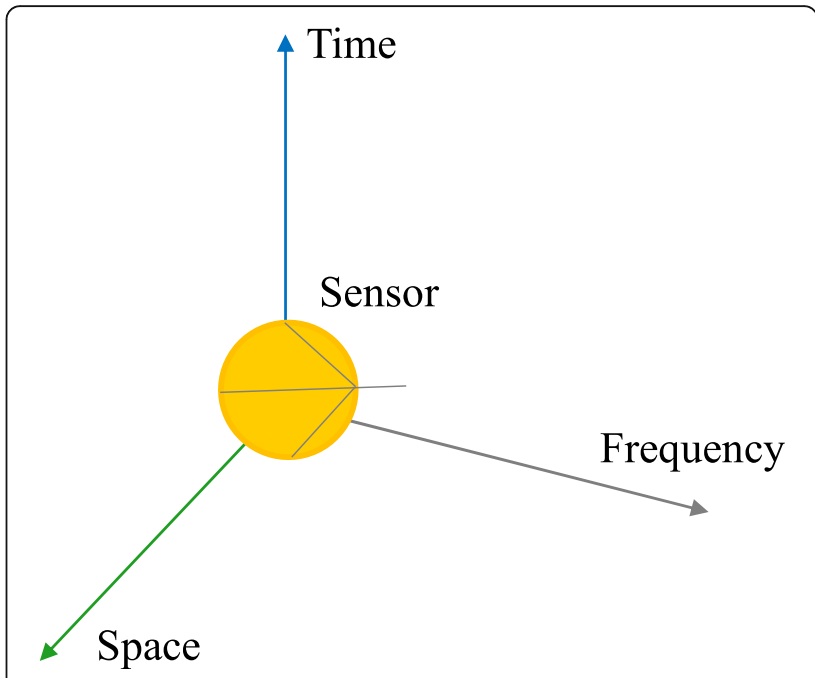

Fig. 1 Sensing node definition in three-dimension domain. Figure shows sensing node definition in three-dimension domain

facts gleaned from the research is that the frequency response can be utilized as a resolution for target description in the dynamic state monitoring. Thereby in the continuous-spatial domain, the sensor with frequency $f$ is transformed to a coordinate-based representation (Fig. 2).

A general tracking target $r_{T}$ in the plane is positioned by $\left(x_{T}, y_{T}\right)$ in Cartesian coordinates. The object in the aforementioned three-dimension domain is delivered as $r_{T}=\left\{t_{T}, s_{T}, f_{T}\right\}$, which can also be expressed as the detection signal $\left(x_{T}, y_{T}\right)$ at time $t_{T}$. In general, a set of properties are required to form the concentration on the spatial domain; the variation scope for detecting is named as $S T$ for target interpretation in this paper. Since the time and spatial features show significant correlation, the frequency within this period is distinguished with three different thresholds $t h_{1}, t h_{2}$, and $t h_{3}$. According to the statistical analysis of our tracking signals, the value $t h_{1}$ is the inflection of frequency trend, at which point

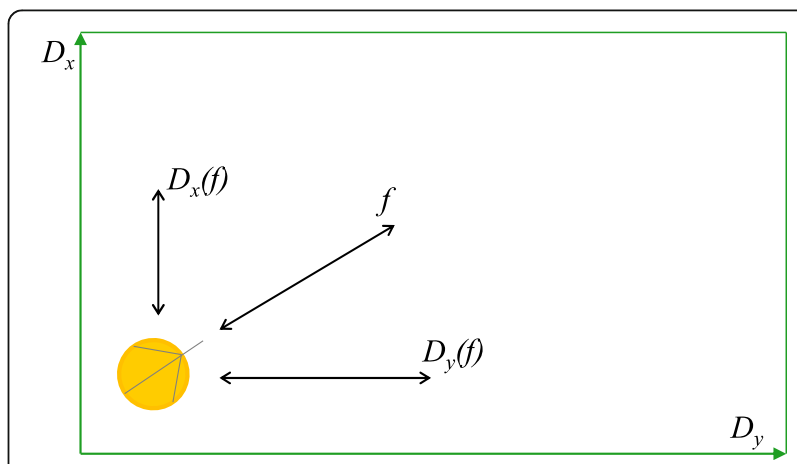

Fig. 2 Spatial domain description of sensor. Figure shows spatial domain description of sensor 
the direction reverses. Meanwhile, there is a frequency shift between $t h_{2}$ and $t h_{3}$. In this way, both of them equal zero. Frequencies between these two can be regarded negative for computation. Figure 3 shows the frequency curve for target property construction in time domain.

The consistency of the tracking target governs the domain transformation. Note that when scanning the object, the target may move from one $S T$ to another. This way, we also obtain a continuous multi-domain target in a quantization form. The derived target with updated representation is written as $r_{S}=\left\{t_{S}, s_{S}, f_{S}\right\}$ and the subscripted variable is the corresponding mark in new region. To extend the sensor detection from one scope to the entire range, the system interference and measurement error should therefore be considered for accurate positioning. Computation with differentiation position data are facilitated by using the following formula on the basic of statistical outcome:

$$
r s=\int_{-f_{T}}^{f_{T}} \phi\left(R^{n}\right) \ln \left(\cos \left(\frac{3 \pi}{2} \omega+\lambda\right)\right) d r
$$

together with

$$
\omega=\delta \pi f
$$

where $\lambda$ stands for the correlation coefficient of spatial with Cartesian coordinate system and $\omega$ is variant of frequency $f$ with respect to angle $\delta$ and $\delta$ is given and keep constant. The multi-domain figure based on classical sensing signal of different orders is discrete in its dimension parameter $R^{n}$. Suppose that there are two independent $S T$ s, i.e., the variability of the object trajectories is calculated by the measuring of the two regions. Let typical frequency threshold $\left\{t h_{1}, t h_{2}, t h_{3}\right\}$ denote the identity of $S T$ with respective

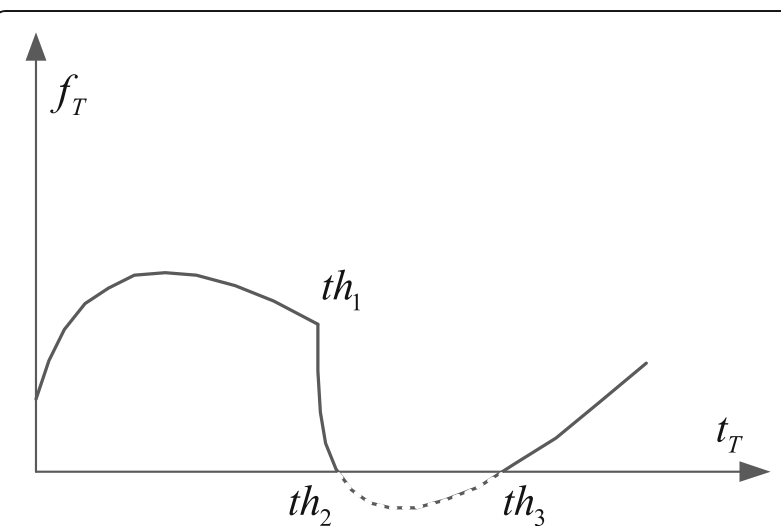

Fig. 3 Frequency variation of target in time domain. Figure shows the frequency curve for target property construction in time domain coefficient $\lambda$. The frequency variation accounting for different $S T$ domains is illustrated in Fig. 4.

The multiple sensors detect the designated target in continuous time series and the sensing frequencies are updated and transformed in a timely manner. Typical tracking parameters are quantified by selecting the proper value of correlation coefficient $\lambda$ for sensing where a wider time interval $\left[t h_{1}, t h_{2}\right]$ and a narrower $\left[t h_{2}, t h_{3}\right]$ are gained due to the increasing of coefficient. The shape of frequency curves in Fig. 4 indicate that, with a same step of tracking, the detection provides a quicker response in $\left[t h_{1}, t h_{2}\right]$ with a $\lambda_{1}$ and in $\left[t h_{2}, t h_{3}\right]$ with a $\lambda_{2}$ where $\lambda_{1} \leq \lambda_{2}$ for computing. Consequently, sensing parameters based on different function scopes can be integrated and further optimized for showing how these values are suitable for tracking purposes. A desired correlation coefficient can be deduced through Eq. 5 .

$$
\lambda=\frac{1}{2} \delta \sqrt[\frac{2}{3}]{\cos ^{2}(\omega t)}
$$

Seeing that $\omega=\delta \pi f$, we shall also define for:

$$
f=\phi(\lambda)
$$

As long as the angle of the angle $\delta$ is fixed, it is thus possible to use the above exhibited formula to determine the domain representation in an effective way.

\section{Target tracking methodology}

\subsection{Tacking overview}

We take the assumption of considering the target addressing as a bivariate resolution issue. The detection method starts by recording the frequency information received from the target. An $S T$ domain identification of the target can be delivered as follows:

$$
M_{d}\left(p_{1}, \cdots p_{N}\right)= \begin{cases}0, & S P_{T}<\lambda \mid f<\phi(\lambda) \\ 1, & S P_{T}>\lambda \mid f<\phi(\lambda)\end{cases}
$$

where $p_{k}$ is signal on the $k$ th dimension and in this research and the maximum $k$ is three. Observing that from Eq. 7, the detection outcome is of discrete value set 0 and 1 . The expression $M_{d}\left(p_{1}, \cdots p_{N}\right)=0$ refers to invalid sensing signals. For the situation $M_{d}\left(p_{1}, \cdots p_{N}\right)$ $=1$, the object is addressed by sensor outputs. The cumulative set of all measurements from sensors collected in time sequences is denoted by $R_{k}, d$ where the subscript $k$ and $d$ are the serial number of sampling vector and $S T$ domains respectively. The track trajectory formulation of a local sensor can be approximated using data statistical analysis 


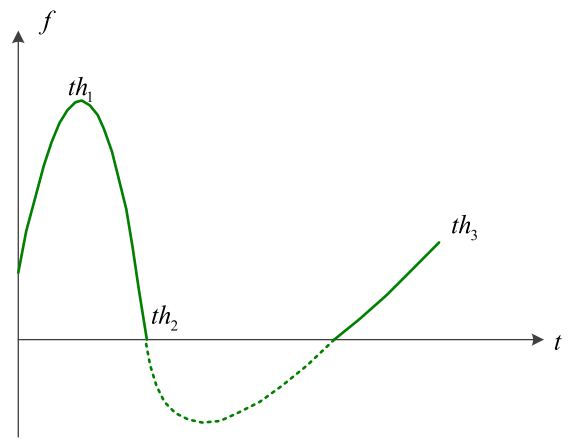

(a) Frequency property with a small $\lambda_{1}$

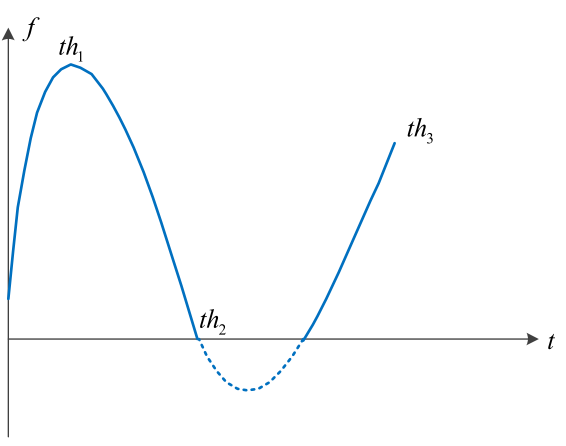

(b) Frequency property with a big $\lambda_{2}$

Fig. 4 Frequency band change with different coefficient. The frequency variation accounting for different ST domains is illustrated in Fig. 4

$$
z\left(p_{1}, \cdots p_{N}\right)=\frac{1}{\delta^{\frac{3}{2}}} \sum_{d=1}^{3}\left\|R_{k, d}\right\|^{\lambda}
$$

In general, two distinguished decision-making mechanisms for target detection are employed [23], which are (1) soft-decision making based on the decoding and fusing of raw sensing data and (2) hard-decision making based on the deployment of multiple parallel sensing elements in communicating condition.

We refer readers to [24-26] for more detailed information on the decision making theory.

For the reason that we consider target tracking using multiple sensors provides better results than single sensor, each local sensor tracker initializes track information and the track information is transmitted for data fusion. Our task is to establish sensing strategy that make the target tracking within the three-dimensional domain using spatial and temporal characteristics. Hardware system with $S T$ principle is made to explore the possibilities for setting up the multi-domain detection and to test algorithms. Signal processing tools are used for data processing, aiming to solve the overlap of the signals coming from the simultaneous sensing devices. After that, techniques for data identification and fusion are implemented and tested.

\subsection{Design of multi-sensing system}

The collection of the data is developed which depends upon the target trajectories as well as the sensor parameters and coordinates. The sensing output is given based on the measurement of frequency and power signals, which are in general finite sequences, properties that is clearly useful for the practical implementation of sensing system $[27,28]$. Considering the statistical characteristics of signal and noise, two estimation parameters detection probability $P_{d}[29,30]$ and false alarm probability $P_{f a}$
$[31,32]$ are introduced to evaluate the working performance in combination with Eq. 7. We have that:

$$
M_{d}\left(p_{1}, \cdots p_{N}\right)=\left\{\begin{array}{l}
0, S P_{T}<\beta \mid f<\phi(\beta) \\
1, S P_{T}>\beta \mid f>\phi(\beta)
\end{array}\right.
$$

where $\beta$ is the correlation threshold of coefficient $\lambda$. As pointed out in Section 2, the tracking evolving according to spatial and temporal characteristics influences the measurement exactly. If we use detection probability and false alarm probability to determine the detecting outcome, we would like to have

$$
\left\{\begin{array}{l}
P_{f a}=\left(M_{d}\left(p_{i}\right)=0\right)=P\left(S P_{T}<\beta \mid f<\phi(\beta)\right) \\
P_{d}=\left(M_{d}\left(p_{i}\right)=1\right)=P\left(S P_{T}>\beta \mid f>\phi(\beta)\right)
\end{array}\right.
$$

We shall thus use a more reliable extension of target demonstration. This extension is the refinement on the foundation of raw frequency and power data. As a consequence, the interpretation $P_{d}$ and $P_{f a}$ are the result of finite number of discrete values in the three-dimensional domain.

$$
\left\{\begin{array}{c}
P_{f a}=e^{-\vartheta(S T)} \sum_{d=1}^{3} \frac{1}{d}\left(\frac{\delta \sqrt[\frac{2}{3}]{\cos ^{2}(\omega t)}}{2 \phi(\lambda)}\right) \\
P_{d}=e^{\frac{-9(S T)}{2 \phi(\lambda)}} \sum_{d=1}^{3} \frac{1}{d}\left(\delta \sqrt[\frac{2}{3}]{\cos ^{2}(\omega t)}\right)
\end{array}\right.
$$

where $\vartheta(S T)$ is the function associated to the frequency and power information in each $S T$. We can then define

$$
\vartheta(S T)=1+\lambda d \sqrt{\frac{\delta}{f}}
$$

Meanwhile, we have indeed $f=\phi(\lambda)$ in line with Eq. 6 . The application of Eq. 11 and Eq. 12 shows the connection of detection accuracy with spatial and temporal properties. If the frequency of a movement increases, 
movement time will be reduced by a factor and the $\vartheta(S T)$ follows the drop. Similarly, if frequency-power function as well as the frequency decreases and the other independent variables remain the same, the detection probability is definitely improved.

Note that the spatial and temporal properties govern the working performances of both hardware and algorithms, especially when the domain basis is given. From sensing output, we obtain that the continuously parameterized $P_{d}$ and $P_{f a}$ will then be defined, corresponding to the devise of hardware and software respectively. The configuration of sensing devices operation principle is shown as follows:

$$
\left\{\begin{array}{c}
P_{f a} \leq P\left(e^{\delta g(k)} \sum_{i=1}^{N} \beta\left|\frac{\delta \cos ^{2}(\omega t+\alpha)}{2 \phi(\lambda)}-\sqrt[\frac{2}{3}]{\cos ^{2}(\omega t)}\right|\right) \\
P_{d} \geq P\left(\sum_{i=1}^{N} \frac{1}{d}\left(\delta \cos ^{2}(\omega t)\right)\right)
\end{array}\right.
$$

Similarly, the programs designing rules can find a feasible optimal solution:

$$
\left\{\begin{array}{c}
P_{f a} \leq P\left(e^{\delta \vartheta(k)} \sum_{d=1}^{3} \sqrt[\frac{2}{3}]{\cos ^{2}(\omega t)} \frac{\delta}{d}\left|1-\frac{1}{2 \phi(\lambda)}\right|\right) \\
P_{d} \geq P\left(\sum_{d=1}^{3} \frac{1}{d}\left(\delta \sqrt[\frac{2}{3}]{\cos ^{2}(\omega t)}\right)\right)
\end{array}\right.
$$

Figures 5 and 6 illustrate the variability of both hard-decision making and soft-decision making of the sensing system during target movement presented above. Figure 5 clearly shows the trend of detection results based on Eq. 13 and Eq. 14. In practical, it is impossible to get a $100 \%$ positioning accuracy with respect to environment noise and system clutter. Compared with soft decision-making scheme, hard decision-making achieves a higher proportion in detection probability as well as a lower proportion in false alarm probability.

In view of the spatial and temporal representation are model depending on the statistic studies; the decision-making strategy is assumed to integrate with it. Further, the working performance of target tracking can be re-estimated. The integrated result is shown in Fig. 6 and the quantitative statistical outcomes confirm the sharp decrease in false alarm probability. Thereupon, we propose a working process which aims at both detecting and interpreting a target from the spatial and temporal characteristics corresponding to the three-dimensional responses, as exhibited in Fig. 7.

The application of target tracking steps is for obtaining a better tracking performance. To start with, the detection scope is divided into $S T$ domains with time series and each sensor involved is made candidate for further utilization. Moreover, the detection frequencies of the target as well as each sensor spatial and time characteristics are computed, based on which, the correlation coefficient of the system is determined. Lastly, the decision-making manner is set while both soft and hard decision-making is corresponding to the $S T$ domain properties. The detection probability and false alarm probability, which represent the detecting accuracy, are

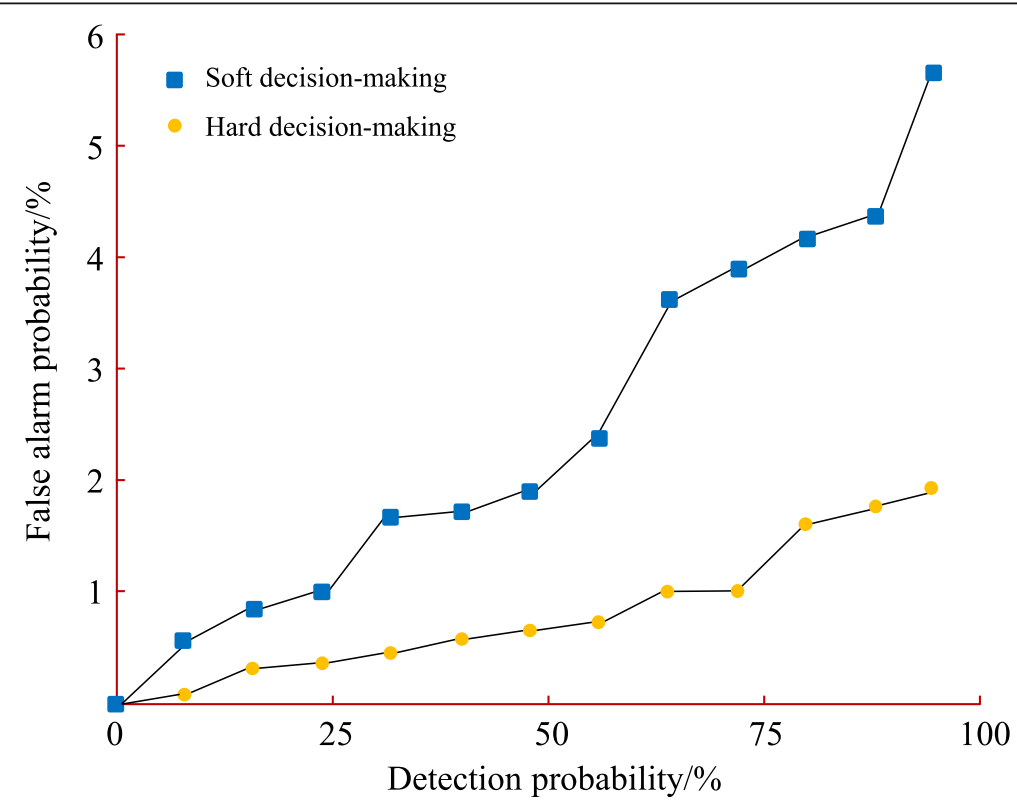

Fig. 5 Decision-making strategies combined with spatial and temporal properties. Figure clearly shows the trend of detection results. The simulation results are shown in Fig. 5 


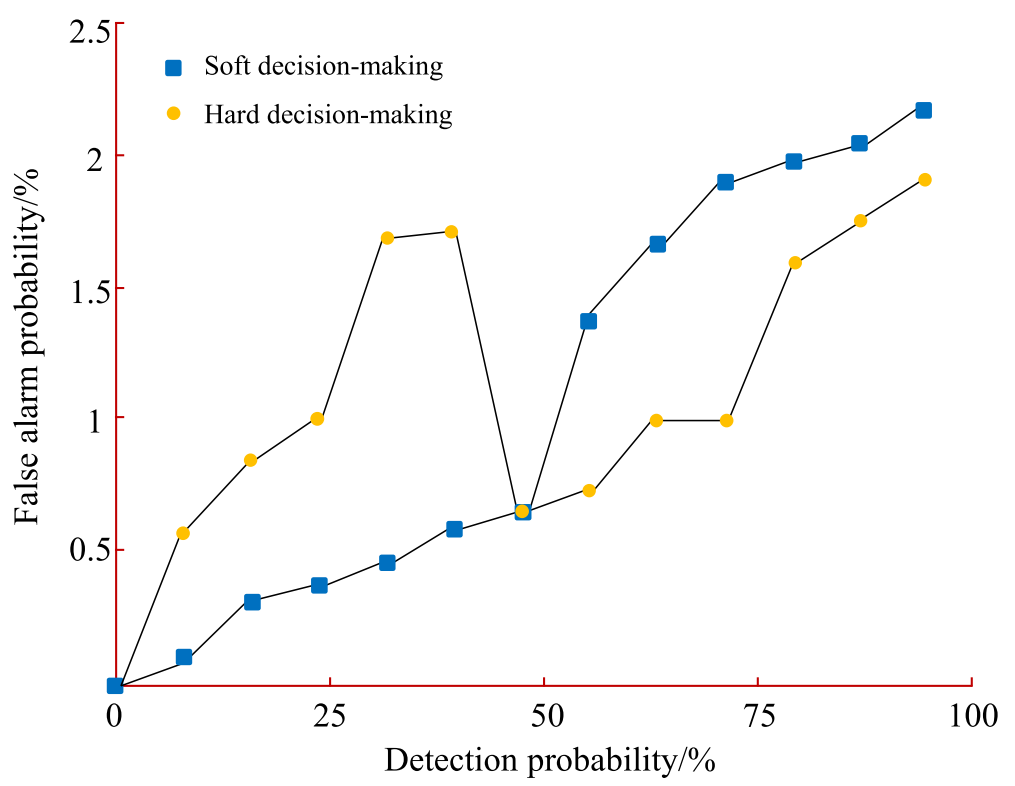

Fig. 6 Comparison of algorithm convergence. The integrated result is shown in Fig. 6

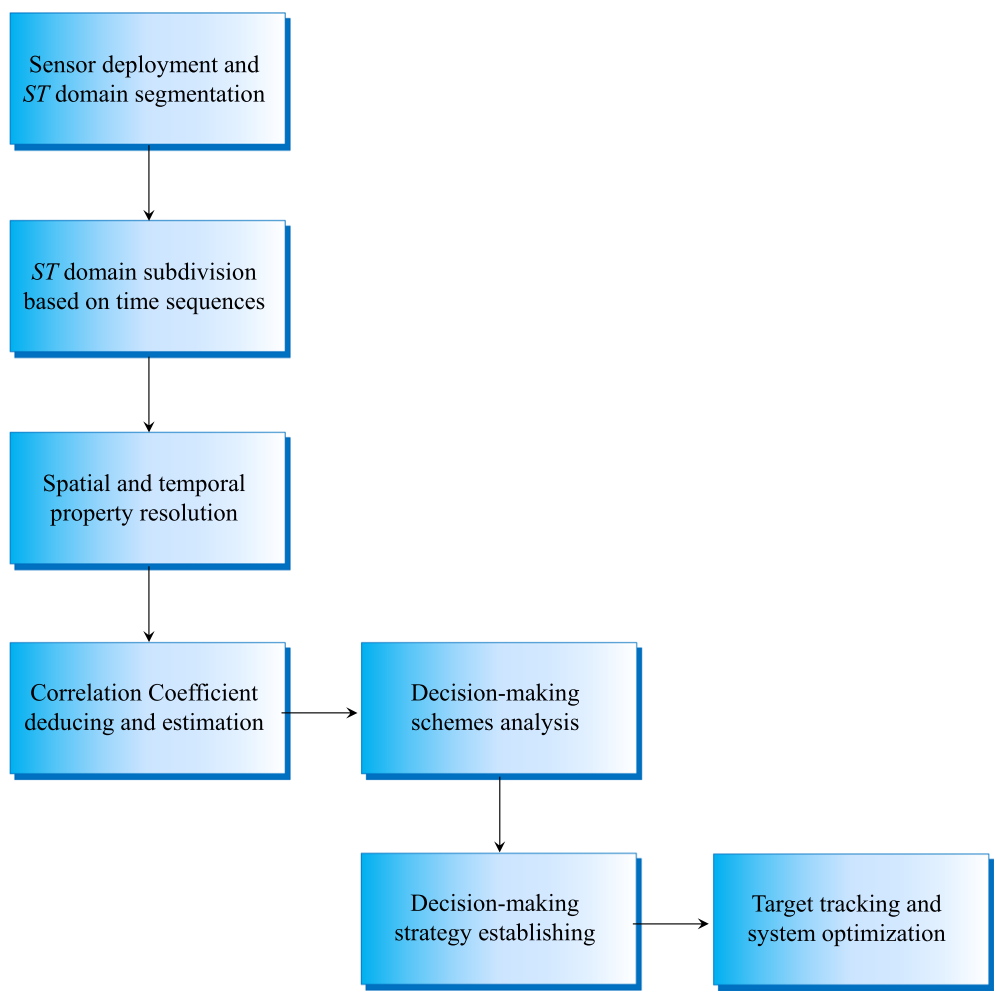

Fig. 7 Block diagram of proposed system working procedure. Both detecting and interpreting a target from the spatial and temporal characteristics corresponding to the three-dimensional responses in Fig. 7 


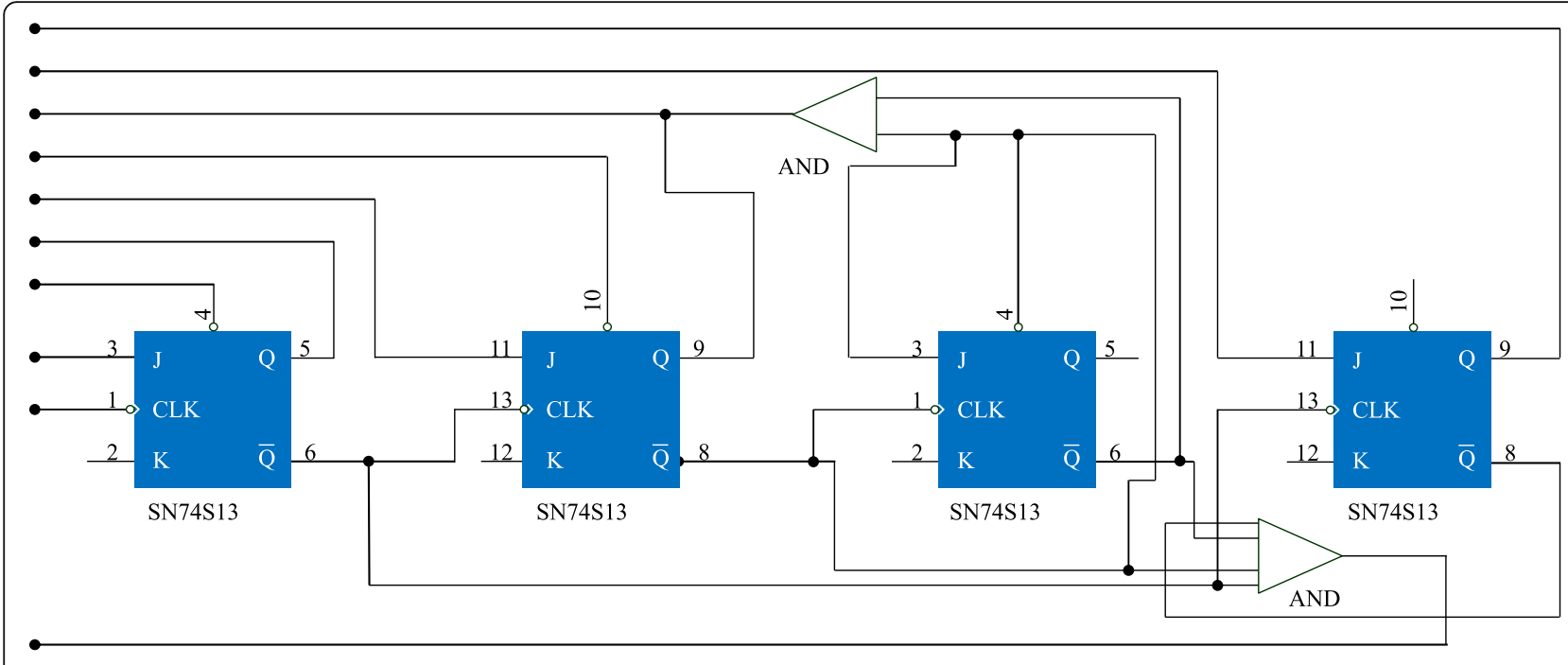

Fig. 8 Circuit diagram of decision-making module

adjusted on the basic of sensing outputs. The outcomes can be viewed as the representation of the decision-making strategy associated to the sensing system. As far as the parameters are regulated, the tracking system is able to find an optimum solution (Fig. 8).

For the purpose of property integration, a chip with the decision-making circuit is designed and installed in the head of the sensor associated to the sensing element. Signal-conditioning circuit with the type of SN74S13 from Texas Instrument is employed for data decoding in in real time [33]. Two and gates are applied for decision making, which both eliminate common mode signal and magnify the system sensitivity. It is worth highlighting that the described circuit is able to work in streaming and hence, no time delay is generated by signal processing.

\section{Experiment}

In order to realize the proposed method in the target tracking, the platform is established. Fundamental experiment is carried out to demonstrate the working performance of actual system through the developed of multi-sensing system. Frequency information is detected and regulated through the sensing element, which can be applied in spatial and temporal characteristic calculation and ST domain segmentation. Meanwhile, the collaboration controlling circuit provides the decision-making function for optimizing the measurement of sensors. Thus, the detection scope of each sensor is re-arranged for obtaining a higher tracking accuracy.

The experiment is conducted in an air-conditioned room with almost constant temperature, humidity, and air pressure. A multi-sensing system with twenty sensors is deployed. One object moving within six $S T$ domains is for tracking. Each $S T$ domain is measured to quantify by time, space, and frequency dimensions. The moving or positioning information of target is recorded from the output of each sensor. The detection sampling rate is $1000 \mathrm{ch} / \mathrm{s}$ with the time series of $10 \mathrm{~min}$. In consideration of such a big amount of data, the detection signal intensity is computed and can be obtained by the following formula exactly.

$$
\left\{\begin{array}{c}
I_{s}=\frac{2 X_{0} \omega_{0}}{1-\frac{f_{0}}{\omega_{0}}} \cos \left(\omega_{t}-\phi_{t}\right) \\
\phi_{t}=\operatorname{arc} \cot \left(\frac{\delta}{\lambda \omega_{0}}\right)
\end{array}\right.
$$

where $\phi_{t}$ is the original domain angle of $S T$.

The decision-making variables are adjusted based on the system outcomes that generate optimum results via domain-feature analysis. In order to statistically evaluate the results, the working performance is shown in Fig. 9. Figure 9a presents the measurement accuracy for three examples. The difference of distinguished determination scheme is statistically significantly with noise added. As long as the clutter is added to the environment, the signal-to-noise ratio is set as $-5 \mathrm{~dB}$ initially. The detection probabilities obtain $80 \%, 70 \%$, and $80 \%$ for hard decision-making scheme, soft decision-making scheme, and proposed decision-making scheme, respectively. The detection accuracy of the three remains increasing whenever the noise declines. Specifically, the proposed determination strategy reaches nearly $99 \%$ when the ratio of signal-to-noise is over 3.

Despite that working accuracy, in general, the optimization of sensor number not always improves results or, if there is an improvement, the final results are 


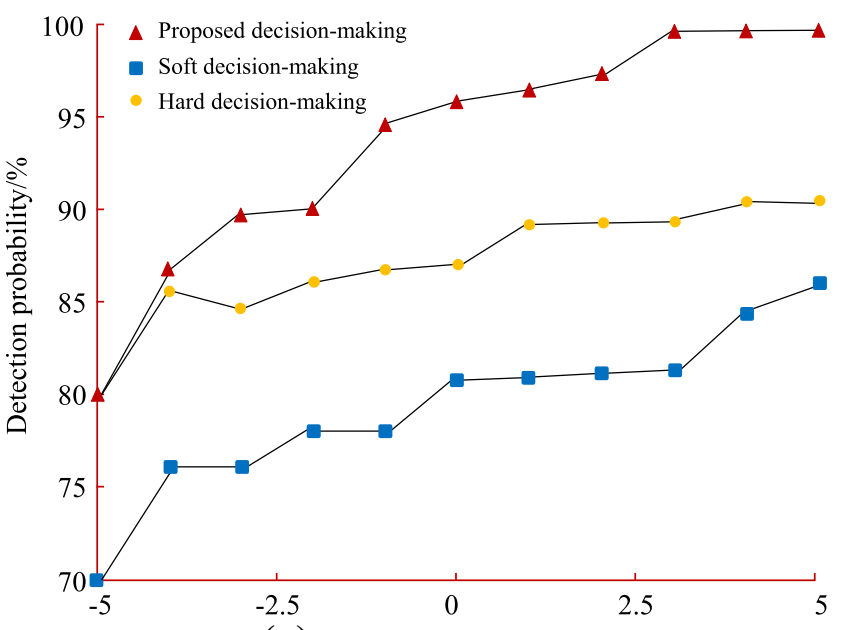

(a) signal-to-noise ratio/dB

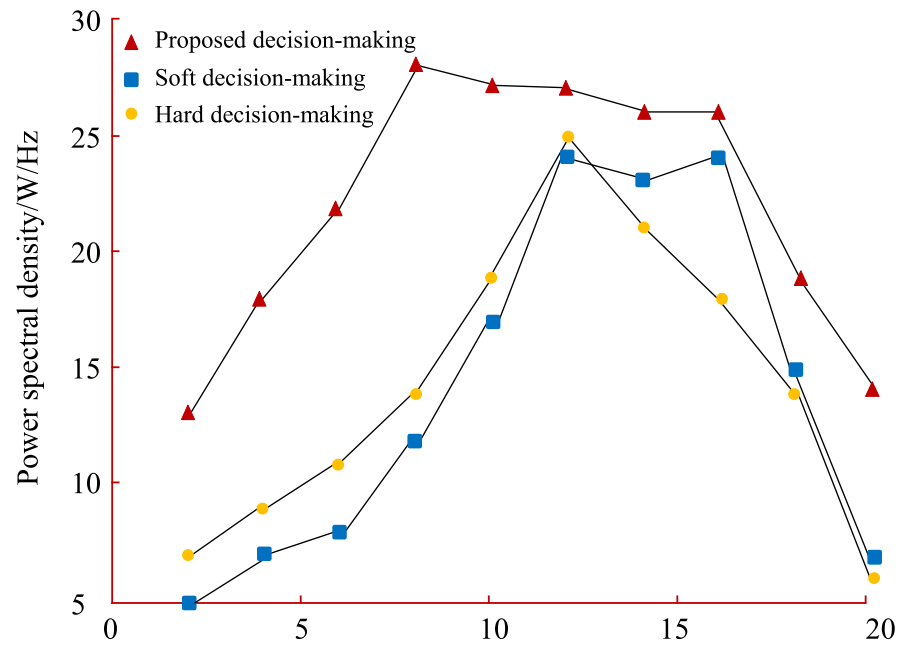

(b) Number of sensors/dimensionless

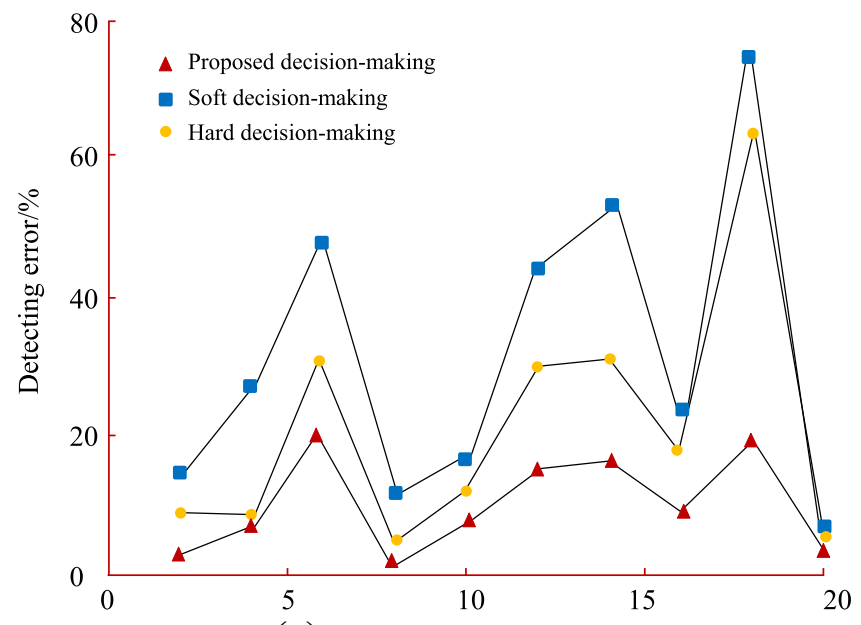

(c) Tracking time/minute

Fig. 9 a Detection accuracy with clutter. b PSD for fixed frequency and different sensor number. c Detection error for different tracking time 
far from being the optimal ones. Figure $9 \mathrm{~b}$ indicates this effect for $1 / f$ signal spectrum. The power spectral density (PSD) is calculated at the same frequency using fast Fourier transformations technique. Maximum PSD points for different decision-making strategies are 12, 12 , and 9 , respectively. This result provides evidence that the configuration of sensing system does influence the working efficiency.

In Fig. 9c, the impact of different time duration on the detection error rate is evaluated. The experiments in this study attempt to conduct 2- to 20-min tests where fluctuation exists for all the three strategies. The best value obtained is at the point of eight tracking minutes using proposed determination strategy, which is $2 \%$ deviation rate. After that, the increase of number obtains counterproductive results.

\section{Conclusions and future work}

This work has presented the multi-sensing determination strategy to track objects from an established scope by using the spatial and temporal characteristics. The decision-making scheme is analyzed in an attentional oriented task. Results indicate that the detection accuracy of the sensing system can be estimated via probability-based functions by segmenting the working region into three-dimensional domain. The proposed domain dividing method as well as decision-making principle presented in this paper improves the detection accuracy and provides optimal configuration of the system development.

Firstly, by replacing the traditional tracking technique for a more efficient one, to improve the working performance of target tracking. This spatial and time property is studied with the collection of tracking frequency. Combined with the real-time performance, the $S T$ domains are generated.

Secondly, the decision-making strategy principle is also regulated. While two types of decision-making strategies (hard decision-making and soft decision making) are employed widely, both of them are considered and analyzed. A more applicable decision-making strategy is proposed. This changed integrated with spatial and temporal feature is utilized in connection with the sensing element as a determination module.

The paper also presented a multi-sensing system set up and optimization process for practical use. In addition, all the basis for system deployment is carefully computed and analyzed statistical analysis.

This study offers an opportunity to the improvement of target tracking accuracy in concerning of the three-dimensional outcome integration. Experiments are conducted on the proposed system to verify its working performance, which is in comparison of two traditional-used methods. The testing results, even if imperfect, provide a direction to explore the tracking properties on the foundation of natural characteristics such as their working frequency.

Future work should pay more attention to the more complex situations where multiple objects are positioned within the tracking scope to explore whether the simple decision-making strategy can also be extended to a multi-target case. Clearly, it seems that the system can precisely segment the region due to the natural characteristic; it is still an open question if the determination strategy could identify each target among all of them.

\section{Abbreviations \\ DMS: Decision-making strategy; MSS: Multi-sensing system; STC: Spatial and temporal characteristics; WSN: Wireless sensor networks}

\section{Acknowledgements}

The authors would like to thank all kinds of funds for funding.

\section{Funding}

This research is supported by the Natural Science Foundation of Hunan Province, China (Grant No. 2018JJ2023) and supported by Shandong Province Education and Science Planned Research Topics during the "12th Five-Year Plan" Special Computer Teaching Project, China (Grant No.YBJ15006).

\section{Availability of data and materials}

Not applicable.

\section{Authors' contributions \\ LY is in charge of the major theoretical analysis, algorithm design, and numerical simulations; the others were in charge of part of the theoretical analysis and algorithm design. All authors read and approved the final manuscript.}

\section{Competing interests}

The authors declare that they have no competing interests.

\section{Publisher's Note}

Springer Nature remains neutral with regard to jurisdictional claims in published maps and institutional affiliations.

\section{Author details}

${ }^{1}$ College of Information and Electronic Engineering, Hunan City University, Yiyang 413000, China. ${ }^{2}$ College of Computer, Heze University, Heze 274000, Shandong, China.

Received: 31 January 2019 Accepted: 25 April 2019

Published online: 10 May 2019

\section{References}

1. M. Kalandros, L.Y. Pao, Multisensor covariance control strategies for reducing bias effects in interacting target scenarios. IEEE Trans. Aerosp. Electron. Syst. 41(1), 153-173 (2005)

2. S. Musick, R. Malhotra, Chasing the elusive sensor manager. Aerospace Electron. Conf. 1, 606-613 (1994)

3. T. Kerr, Modeling and evaluating an empirical INS difference monitoring procedure used to sequence SSBN NAVAID fixes. Navigation 28(4), 263-285 (1981)

4. C. Lundquist, L. Hammarstrand, F. Gustafsson, Road intensity based mapping using radar measurements with a probability hypothesis density filter. IEEE Trans. Signal Process. 59(4), 1397-1408 (2011)

5. J.S. Wasson, J.R. Sturdevant, D.M. Bullock, Real-time travel time estimates using media access control address matching. ITE J. 78(6), 20-23 (2008)

6. S. Young, in University of Maryland. Bluetooth traffic monitoring technology — concept of operation \& deployment guidelines. Dissertation (2008) 
7. J.A. Kirkup, D.D. Rowlands, D.V. Thiel, Team player tracking using sensors and signal strength for indoor basketball. IEEE Sensors J. 16(11), 4622-4630 (2016)

8. P.C. Liang, P. Krause, Real-time indoor patient movement pattern telemonitoring with one-meter precision, Eai International Conference on Wireless Mobile Communication and Healthcare (2014), pp. 141-144

9. G. Tang, X. Liu, C. Chen, et al., Active tracking using color silhouettes for indoor surveillance, International Conference on Wireless Communications and signal processing (2015), pp. 1-5

10. B.T. Vo, C.M. See, N. Ma, W.T. Ng, Multi-sensor joint detection and tracking with the Bernoulli filter. IEEE Trans. Aerosp. Electron. Syst. 48(2), 1385-1402 (2012)

11. A. Yaeli, P. Bak, G. Feigenblat, et al., Understanding customer behavior using indoor location analysis and visualization. IBM J. Res. Dev. 58, 3):1-3)12 (2014)

12. F. De Cillis, F. De Simio, L. Faramondi, et al., Indoor positioning system using walking pattern classification, Mediterranean Conference of Control and Automation (2014), pp. 511-516

13. E.H. Lee, T.L. Song, Multi-sensor track-to-track fusion with target existence in cluttered environments. IET Radar Sonar Navig. 11(7), 1108-1115 (2017)

14. H. Ken, Managing sensor performance uncertainty in a multi-sensor robotic system. Dissertation (University of South Florida, Tampa Bay, 1994)

15. V. Zadorozhny, Y.F. Hsu, in Scalable Uncertainty International Conference on Scalable Uncertainty Management: Scalable Uncertainty Management. Conflict-aware historical data fusion (2011), pp. 331-345

16. M.H. Habaebi, R.O. Khamis, A. Zyoud, M.R. Islam, RSS based localization techniques for ZigBee wireless sensor network, International Conference on Computer \& Communication Engineering (2015), pp. 72-75

17. L. Matthies, S.A. Shafer, Error modeling in stereo navigation. IEEE J. Robot. Autom. 3(3), 239-248 (1987)

18. A.S. Housfater, Sequential Monte Carlo methods for multi-sensor tracking with applications to radar systems. Dissertation (Ryerson University, Toronto, 2006)

19. S. Deb, M. Yeddanapudi, K. Pattipati, Y. Bar-Shalom, A generalized S-D assignment algorithm for multisensor-multitarget state estimation. IEEE Trans. Aerosp. Electron. Syst. 33(2), 523-538 (1997)

20. A. Belmonte Hernandez, G. Hernandez Penaloza, F. Alvarez, G. Conti, Adaptive fingerprinting in multi-sensor fusion for accurate indoor tracking. IEEE Sensors J. 17(15), 4983-4998 (2017)

21. J.F. Soechting, Effect of target size on spatial and temporal characteristics of a pointing movement in man. Exp. Brain Res. 54(1), 121-132 (1984)

22. G. Sapiro, A. Cohen, A.M. Bruckstein, A subdivision scheme for continuousscale B-splines and affine-invariant progressive smoothing. J Math Imaging Vision 7, 23-40 (1997)

23. Y. Cui, R.M. Voyles, J.T. Lane, A. Krishnamoorthy, M.H. Mahoor, A mechanism for real-time decision making and system maintenance for resource constrained robotic systems through ReFrESH. Auton. Robot. 39(4), 487-502 (2015)

24. D. Zhang, A joint response model for matched decision makers: exploring decision making mechanism for mutually-selected agents. Dissertation (Rensselaer Polytechnic Institute, Troy, 2016)

25. D.-W. Yue, H.H. Nguyen, Orthogonal DF cooperative relay networks with multiple-snr thresholds and multiple hard-decision detections. EURASIP J. Wirel. Commun. Netw. (2010). https://doi.org/10.1155/2010/169597

26. J.-T. Sung, H.-T. Pai, B.-H. Lee, Performance analysis for distributed classification fusion using soft-decision decoding in wireless sensor networks, EUC 2007 Embedded and Ubiquitous Computing (2007), pp. 623-634

27. M.H. Chen, P.F. Yan, A multiscale approach based on morphological filtering. IEEE Trans Pattern Anal Mach Intell 11(7), 694-700 (1989)

28. R. Pokrywka, Reducing false alarm rate in anomaly detection with layered filtering, International Conference on Computational Science (2008), pp. 396-404

29. J. Naganawa, H. Miyazaki, H. Tajima, Detection probability estimation model for wide area multilateration, Integrated Communications, Navigation and Surveillance Conference (2017), pp. 2B1_1-2B1_15

30. Q. Zheng, R. Yang, Z. Shan, J. Chen, Research on the detection probability of airdrop torpedo based on analytical method, International Conference on Progress in Informatics and Computing (2016), pp. 674-678

31. Y. Wang, Y. Zhang, Q. Zhang, S. Wu, Optimal selection of false alarm probability for dynamic spectrum access. IEEE Commun. Lett. 17(5), 844-847 (2013)

32. L. Anitori, M. Otten, P. Hoogeboom, False alarm probability estimation for compressive sensing radar, IEEE Radar Conference (2011), pp. 206-211

33. SN74S138A: 3-Line To 8-Line Decoders/ Demultiplexers datasheet, http:// www.ti.com/product/SN74S138A/technicaldocuments?keyMatch= 74S13\&tisearch=Search-EN-Products

\section{Submit your manuscript to a SpringerOpen ${ }^{\circ}$ journal and benefit from:}

- Convenient online submission

- Rigorous peer review

- Open access: articles freely available online

High visibility within the field

- Retaining the copyright to your article

Submit your next manuscript at $\boldsymbol{\nabla}$ springeropen.com 